\title{
Relation between abnormal synergy and gait in patients after stroke
}

\author{
Kaoru Sakuma ${ }^{1,2^{*}}$, Koji Ohata ${ }^{1}$, Keisuke Izumi ${ }^{3}$, Yu Shiotsuka ${ }^{4}$, Tadashi Yasui ${ }^{5}$, Satoko Ibuki ${ }^{1}$ and Noriaki Ichihashi ${ }^{1}$
}

\begin{abstract}
Background: The abnormal synergy seen in patients after stroke is considered to limit the ability of these patients. However, in the lower extremity, antigravity torque generation rather than precise movement is needed for functions such as sit-to-stand movement and gait. Therefore, the ability to generate torque may be important either as a primary movement or as an abnormal synergy. We attempted to quantify the torque generation in the lower limb, selectively and as an abnormal synergy, and its relation with gait.

Methods: Selectively generated plantar flexion torque in the ankle and plantar flexion torque secondarily generated accompanying maximal hip extension (i.e., torque generated with abnormal synergy) were measured in subjects after stroke and control subjects. In subjects after stroke, secondary torque generation while controlling hip extension torque as $25 \%, 50 \%$, and $75 \%$ of the maximal hip extension was also measured. The relation of torque generation with the gait speed and timed-up-and go test (TUG) was also analyzed.
\end{abstract}

Results: In subjects after stroke, there was no difference between the amount of plantar flexion torque generated secondarily and the selectively generated torque, whereas the selective torque was significantly greater in control subjects. Pearson product-moment correlation coefficient analysis revealed that TUG speed is related to secondarily generated torque accompanying maximal hip extension but not with selectively generated torque.

Conclusion: Secondarily generated torque was found to be a factor that affects TUG speed, and the ability to generate torque even through abnormal synergy may help for gait ability in subjects after stroke.

Keywords: Abnormal synergy, Gait, Stroke

\section{Background}

After stroke, many patients experience motor impairments [1] that cause gait deficits [2-7]. The ability to perform activities of daily living depends on gait ability [2]. Therefore, it is important to understand the relation between impairment and gait deficit in patients after stroke.

Abnormal synergy is a motor impairment in patients after stroke $[8,9]$. Some patients lose independent control of selected muscle groups, resulting in coupled joint movements that are often inappropriate for the desired task. These coupled movements are known as abnormal

\footnotetext{
* Correspondence: sakuma.kaoru.27u@st.kyoto-u.ac.jp

'Department of Physical Therapy, Human Health Sciences, Graduate School of Medicine, Kyoto University, 53, Kawahara-cho, Shogoin, Sakyo-ku, Kyoto 606-8507, Japan

${ }^{2}$ Japan Society for the Promotion of Science, 5-3-1 Kojimachi, Chiyoda-ku, Tokyo 102-0083, Japan

Full list of author information is available at the end of the article
}

synergy. For the lower limb, abnormal synergy is grouped into extension synergy (internal rotation, adduction, and extension of the hip; extension of the knee; and extension and inversion of the ankle) and flexion synergy (external rotation, abduction, and flexion of the hip; flexion of the knee; and flexion and eversion of the ankle) [9]. Abnormal synergy has been recognized as a factor limiting the motor rehabilitation of patients after stroke [10-14]. Studies measuring the relative phase in stroke patients have shown that patients after stroke differ from normal subjects in intra- [15-17] and inter-limb [18] coordination of the lower limb. Difference in kinematics is also seen in stroke subjects [19]. Many patients recover gait activity with compensatory adaptation [19-22]. A previous study showed that the Fugl-Meyer synergy score was significantly correlated with gait speed [23]. It was also found that attempts to make gait patterns resemble those of neurologically healthy adults by using Lokomat or a 
robotic knee orthosis did not result in the extinction of abnormal synergy $[24,25]$. The ability to exert lower-limb muscle torque is important for gait $[3,6,26,27]$. By quantitatively assessing the abnormal synergy in the lower limb by measuring the torque exerted as abnormal synergy, and then investigating its relation with gait ability, we may be able to gain insight into the relation between abnormal synergy and gait ability. In a study quantifying the upperlimb abnormal synergy in patients after stroke, Dewald et al. $[28,29]$ measured the joint torque that the subjects were attempting to maximize as the primary torque and the torques at other directions as secondary torques, along with electromyographic (EMG) measurements during voluntary isometric muscle contractions. They found that patients after stroke had significantly decreased maximum voluntary torque compared with the control subjects, and that the patients exhibited reductions in maximum torques when required to control the secondary torque $[28,29]$. Patients after stroke could not generate voluntary torque selectively without generating torque in other joints. Other studies that applied a similar method to the lower limb $[13,14]$ suggested that the primary contributor to lower-limb motor deficits was the weakness of voluntary torque rather than the value of abnormal synergy.

Patients after stroke lack the ability to selectively generate voluntary torque without generating joint torque in other directions and in other joints both in the upper and lower limbs. We hypothesized that abnormal synergy in the lower limb may compensate for the weakness of agonist muscle in patients after stroke. Furthermore, we hypothesized that if the previous hypothesis is correct, a relation between abnormal synergy of the lower limb and gait would also be observed. However, the relation between of the lower limb and gait has not yet been clarified.

To study the abnormal synergy of the lower limb and its relation with gait, we measured the ankle joint torque generated secondarily during maximum voluntary isometric hip extension (secondary torque, STo) and the maximum voluntary ankle plantar flexion torque primarily generated without hip extension torque generation (primary torque, PTo). Then, we investigated whether PTo and STo are related to the gait speed or TUG in patients after stroke.

\section{Methods}

\section{Subjects}

Eleven subjects after stroke and 13 control subjects participated in this study. The characteristics of the subjects are summarized in Table 1.

The subjects were recruited in the community through convenience sampling. The subjects after stroke satisfied the following criteria: (i) hemiparesis resulting from a unilateral cortical or sub-cortical brain lesion; (ii) $>6$ months since onset; and (iii) able to walk in the community. The
Table 1 Characteristics of the subjects

\begin{tabular}{lll}
\hline & $\begin{array}{l}\text { Subjects after stroke } \\
(\mathbf{n = 1 1 )}\end{array}$ & $\begin{array}{l}\text { Control subjects } \\
(\mathbf{n = 1 3 )}\end{array}$ \\
\hline Age (years) & $51.1 \pm 10.7$ & $51.1 \pm 9.6$ \\
Sex (n): M/F & $8 / 3$ & $13 / 0$ \\
Height (cm) & $164.5 \pm 7.4$ & $169.0 \pm 6.3$ \\
Weight (kg) & $60.3 \pm 8.7$ & $67.6 \pm 10.9$ \\
B.R.S. (n): III/IV/N/NI & $2 / 4 / 3 / 2$ & \\
Time since onset (months) & $90.7 \pm 79.9$ & \\
Paretic side (n): R/L & $7 / 4$ & \\
\hline
\end{tabular}

$F$, female; $M$, male

$R$, right; $L$, left.

Values are presented as means \pm standard deviation (SD), unless

otherwise indicated.

exclusion criteria for the subjects after stroke were as follows: (i) history of other neurologic, respiratory, cardiovascular, or orthopedic problems that influence their participation in the study; (ii) brainstem or cerebellar lesions; and (iii) inability to provide informed consent. Control subjects with similar age to the subjects after stroke were recruited. Control subjects were excluded if they had neurologic, respiratory, cardiovascular, or orthopedic problems. All subjects provided informed consent before participation in this study. This study was approved by the institutional review board of Kyoto University.

\section{Primary torque and secondary torque}

A schematic of the test set-up is shown in Figure 1. Measurements were taken for all subjects in the supine position (hip extension angle of $0^{\circ}$, knee extension angle of $0^{\circ}$, and ankle dorsiflexion angle of $0^{\circ}$ ). The measured side was the paretic lower limb for subjects after stroke and the right limb for control subjects.

For recording the PTo and STo, an originally developed orthosis-type device with a load cell that could measure the ankle plantar flexion torque (Gait Solution Design; Kawamura Gishi, Osaka, Japan [30]) was used. A load cell was inserted above the hydraulic cylinder in the oil damper unit. Upon the generation of the plantar flexion torque by the subject, the hydraulic cylinder pushed the load cell as a counterforce; the counterforce, which reflects the planter flexion torque, was numerically indicated in a personal computer linked the device [30]. For recording the hip extension torque, a handheld dynamometer ( $\mu$-tas F-1; ANIMA Corp., Tokyo, Japan) was used. The subject's foot was attached to the orthosis-type device and the thigh was attached to the handheld dynamometer. The PTo and the STo during maximum voluntary hip extension torque were each measured once in both the control subjects and the subjects after stroke. The subjects started in a relaxed state and slowly increased the torque generation to the maximum level, which was held for approximately $3 \mathrm{~s}$. 


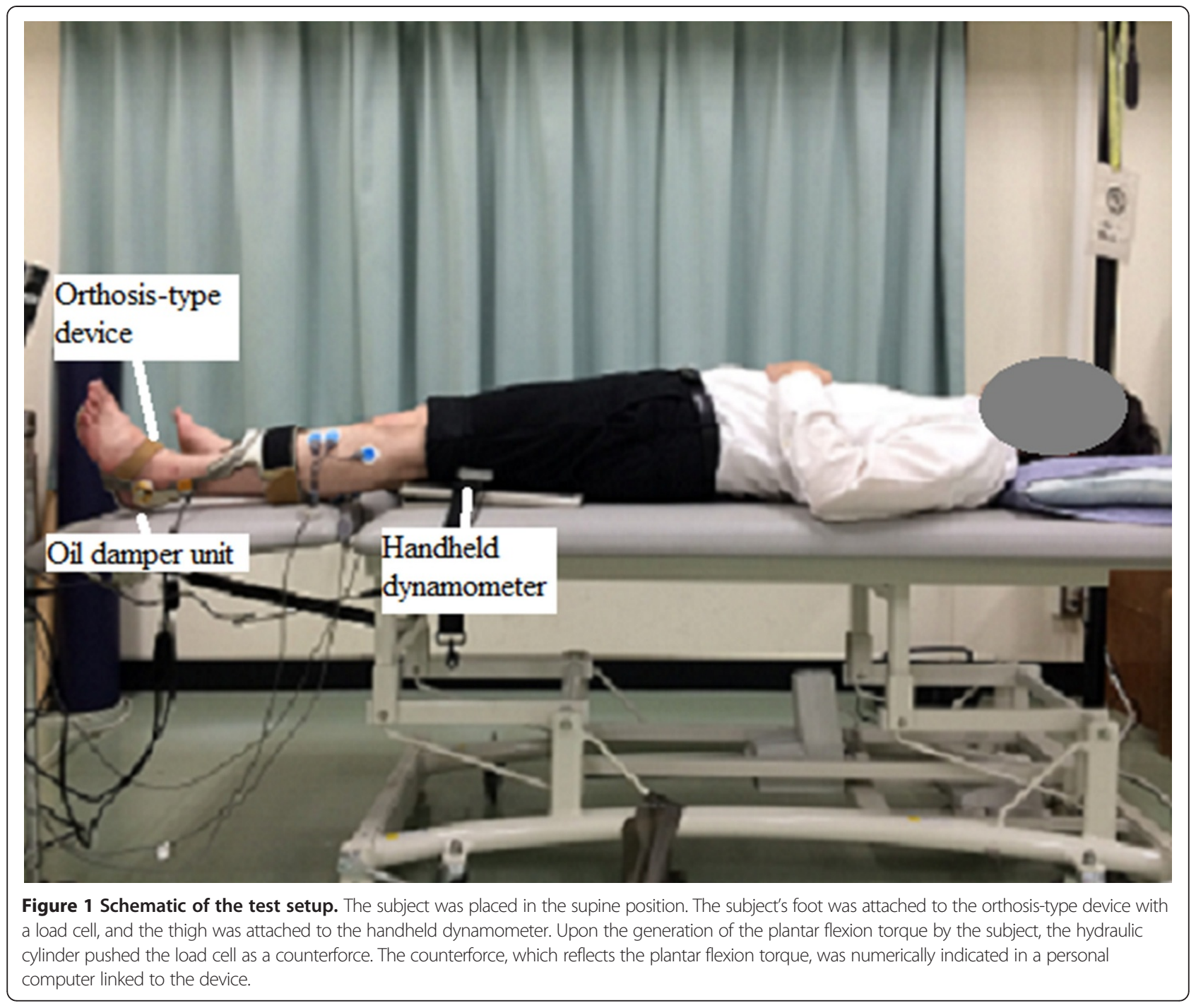

In the subjects after stroke, secondary torque generation while controlling hip extension torque as $25 \%, 50 \%$, and $75 \%$ of the maximal hip extension (25\%STo, $50 \%$ STo, and $75 \% \mathrm{STo}$, respectively) was also measured in a random order. The subjects were provided with visual feedback about their exerted hip extension torque, measured using the handheld dynamometer, to aid them in exerting a force matching the target torque. The subjects practiced two to three times until they could control their hip extension torque at the target value. The ankle plantar flexion torque was measured once for each target torque for $3 \mathrm{~s}$ where the generated hip extension torque matched the target torque.

The plantar flexion torque collected in each trial was computerized concurrently with the EMG data. The ensemble ankle plantar flexion torque in the 500-ms window was normalized to body weight $(\mathrm{Nm} / \mathrm{kg})$.

The EMG recordings were collected by using the Telemyo $2400 \mathrm{~T}$ hardware (Noraxon USA Inc., Scottsdale,
Arizona, USA; sampled at $1500 \mathrm{~Hz}$, band-pass filter at 10-500 Hz). Bipolar surface electrodes (Blue Sensor; Medicotest Inc., Olstykke, Denmark) with an interelectrode distance of $20 \mathrm{~mm}$ (center-to-center) were placed on the muscle belly of the tibialis anterior, gastrocnemius (on the lateral head), and soleus muscles. Electrode placement on the tibialis anterior was at one-third on the line $1-2 \mathrm{~cm}$ lateral to the tibia; the gastrocnemius, at one-third on the line between the head of the fibula and the heel; and the soleus, at one-half to two-third on the line between the head of the medial condyles of the femur and the tip of the medial malleolus. A ground electrode was placed on the head of fibula. We prepared the electrode locations by cleaning the sites with alcohol wipes. The EMG data were smoothed by using a root-mean-square algorithm over a 10 -ms window, and the mean value for 1-s duration was taken concurrently with the maximum ankle plantar flexion torque generation. 


\section{Gait}

The 10-m gait speed and TUG were measured in the subjects after stroke. In the 10-m gait test and TUG, the subjects wore an ankle foot orthosis that allowed ankle movement; however, they did not use a cane or any other walking aids. For recording the gait speed, the subjects after stroke were asked to perform gait trials twice at their preferred speed. The gait speed $(\mathrm{m} / \mathrm{s})$ was calculated from the mean of the $10-\mathrm{m}$ gait time data. For recording the TUG, the time taken to stand up from a seated position, walk forward for $3 \mathrm{~m}$, walk back, and then return to the seated position was measured once.

\section{Data analysis}

One of the characteristics of abnormal synergy recognized as the limiting factors for the motor rehabilitation was that abnormal synergy becomes more prominent when the subjects attempt to generate higher joint torques $[8,9]$. To assess whether these characteristics is reflected in the $25 \% \mathrm{STo}, 50 \% \mathrm{STo}, 75 \% \mathrm{STo}$, and STo, repeated-measures analysis of variance was used to analyze the effect of the exertion condition of hip extension on the ankle plantar flexion torque. Tukey's test was used to examine the subsequent planned comparisons between each exertion condition.

Second, two-factor analyses of variance and t-test were used to analyze the difference between the group factor (subjects after stroke and control) and the exertion condition factor (PTo and STo) in the plantar flexion torque. The $t$ test was used to analyze the difference between PTo and STo on EMG.

Finally, Pearson product-moment correlation coefficient was used to assess the relation between the gait speed or TUG and PTo or STo. Then, stepwise multiple linear regression analysis was used to assess the influence of PTo and STo on gait speed or TUG.

A significance level of 0.05 was used for all comparisons.

\section{Results}

Relation between secondary torque generation and percent hip extension torque in the subjects after stroke

The correspondence of secondary plantar flexion torque with percent hip extension torque is summarized in Figure 2. There was a significant main effect of exertion condition $(\mathrm{p}<0.01)$. The $50 \%$ STo was higher than the $25 \%$ STo, and the STo was higher than the $75 \%$ STo.

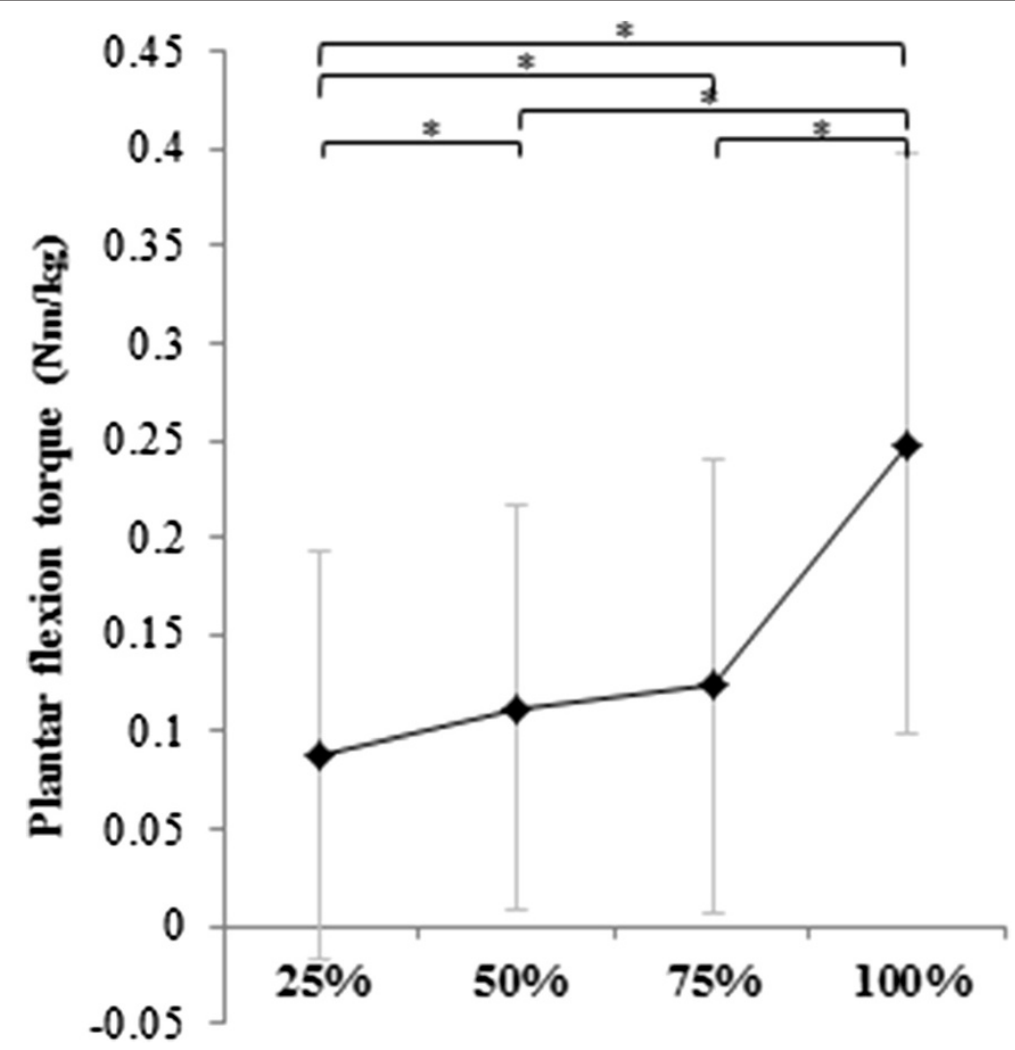

Figure 2 Correspondence of secondary plantar flexion torque with percent hip extension torque in subjects after stroke. An asterisk (*) denotes statistical significance between conditions $(p<0.05)$. The figure indicates the plantar flexion torque (standardized by body weight) generated when exerting the hip extension torque at 25\%,50\%,75\%, and $100 \%$ of the maximal hip extension. There was a significant main effect of exertion condition. The secondarily generated plantar flexion torque (STo) corresponding to $50 \%$ of the maximum voluntary hip extension torque (50\%STo) was higher than the 25\%STo, and the 100\% (STo) was higher than the 75\%STo, 50\%STo, and 25\%STo. The 75\%STo was higher than the 25\%STo. 
Characteristics of primary torque and secondary torque in the subjects after stroke and the control group

Interaction effects were found between the group factor and the exertion condition factor $(\mathrm{p}<0.01)$. The subjects after stroke $(0.1 \pm 0.1)$ showed lower values than the controls in the PTo $(0.6 \pm 0.4, \mathrm{p}<0.05)$, but not in the STo (subjects after stroke, $0.2 \pm 0.2$; controls, $0.2 \pm 0.2$ ). The PTo was significantly higher than the STo in the control group $(\mathrm{p}<0.01)$ but not in the subjects after stroke.

The EMG data during PTo and STo are summarized in Figure 3. The gastrocnemius and soleus activities during PTo were higher than those during STo in the control group but not in the subjects after stroke. The tibialis anterior activity during the STo was higher than during PTo in the subjects after stroke but not in the control group.

\section{Gait}

There were no relation between gait speed and the PTo $(r=0.4)$ or the STo $(r=0.5)$. There was a significant relation between TUG and the STo $(r=0.7)$ but not between TUG and the PTo $(r=0.5)$. Stepwise analysis revealed that the STo was a determinant of TUG $\left(R^{2}=0.5\right)$.

\section{Discussion}

The results of this study revealed that in subjects after stroke, the increase in the percentage of maximum hip extension torque generated by the subjects increased the $\%$ STo. The subjects after stroke exhibited lower torque than the control group in the PTo but not in the STo. The PTo was significantly higher than the STo in the control group but not in the subjects after stroke. Our hypothesis was supported by the observation that there is a correlation between TUG and the STo in the subjects after stroke, and the principal finding of this study is that the STo was the determinant of TUG, as revealed by stepwise analysis. This is the first study that quantitatively demonstrated the inability to selectively generate voluntary torque in the lower limb, and showed that STo affects TUG in patients after stroke.

\section{Relation between secondary torque generation and} percent hip extension torque in the subjects after stroke Controlling agonist activity is a fundamental function required in activities of daily living. Therefore, in this study, we chose a task that requires generating a certain percentage of maximum voluntary torque. The subjects after stroke showed explicit increase in the \%STo as the percentage of required hip extension increased, which is consistent with the characteristics of abnormal synergy described in previous studies $[8,9]$. Therefore, the STo measured in this study is considered to reflect the feature of abnormal synergy.

Characteristics of primary torque and secondary torque in the subjects after stroke and the control group

In a previous study that measured the secondary torque in the ankle joint during maximum voluntary hip extension in both controls and subjects after stroke, the secondary torque was seen in both groups and no differences were found in the rate of the secondary torque to maximum

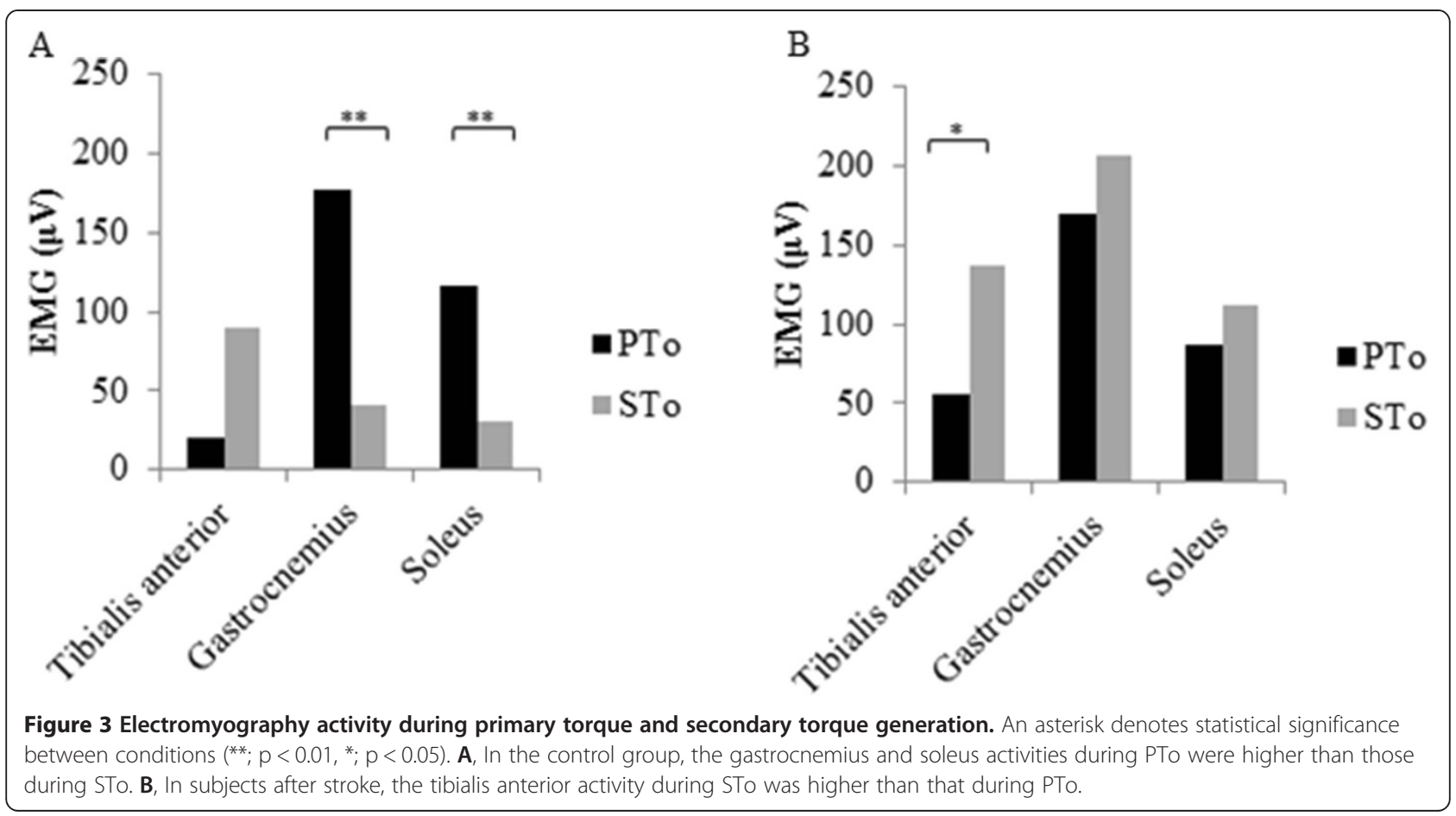


voluntary ankle plantar flexion torque between the groups [13]. Similarly in this study, there were concurrent ankle plantar flexion torques measured as the STo during the generation of the maximum voluntary hip extension torque in both the controls and the subjects after stroke, and there were no differences between the controls and subjects after stroke in the STo torque normalized to body weight. In the current study, the control group could generate considerably higher PTo than STo, whereas the subjects after stroke could only generate PTo torque equivalent to their STo torque. Moreover, the gastrocnemius and the soleus, which are the agonist muscles in ankle plantar flexion, were more activated during STo than during PTo in the subjects after stroke; the opposite was observed in the controls. This is probably due to the disorganization of motor unit recruitment, rate modulation patterns [31,32], antagonist muscle weakness [33], and the abnormal corticospinal responses [34-36] that might affect the contribution of agonist activity to the voluntary torque seen in the subjects after stroke. It should also be noted that the tibialis anterior muscle activity during STo was higher than that during PTo in the subjects after stroke. The co-activation of the antagonist muscle, i.e., the tibialis anterior muscle might have inhibited the generation of plantar flexion torque as STo. However, the gastrocnemius and soleus activities during STo were not higher than those during PTo. Therefore, we conclude that the tibialis anterior muscle activity did not affect the results of this study. In the subjects after stroke, because of the inability to selectively activate the agonist muscle, the STo becomes relatively higher than the PTo.

\section{Correlation with gait ability}

The stepwise analysis revealed that the STo, and not the PTo, was the determinant of TUG. Although there was no significant relation between the STo and gait speed, the correlation coefficient was high. Therefore, we consider that there might be a relation between the STo and gait ability. In previous studies, the relation between the Brunnstrom recovery stage [19] or the Fugl-Meyer assessment [23] and gait speed was reported. However, because these clinical assessments evaluated both recovery from abnormal synergy and improvement of voluntary movement, it was unclear whether abnormal synergy or voluntary movement was related to gait speed. In this study, by quantifying the abnormal synergy measuring the joint torque, the relation between abnormal synergy and gait was revealed for the first time, showing that STo was the determinant of TUG. On the other hand, a previous study showed that the paretic ankle plantar flexion torque was correlated with gait speed in the patients after stroke [27]. We consider that because TUG consists not only of gait but also of sit-to-stand movement, which requires a large torque
[37,38], abnormal synergy might be a related factor. Our results suggest that in subjects after stroke, STo might be adopted to compensate for the inability to generate the voluntary torque during gait.

\section{Limitation}

Three limitations of this study need to be mentioned. First, the PTo and STo measured in this study might be affected by other factors such as co-activation and spasticity. Nevertheless, we could at least assess one aspect of abnormal synergy quantitatively as the joint torque generated concurrently with the intended voluntary torque.

Second, the subjects after stroke recruited in our study were community-dwelling, able to walk. Therefore, our results may not be applicable to patients in a more severe condition after stroke who are unable to live in the community or have greater disability in gait.

Finally, this study did not evaluate abnormal synergy during gait, or the relation of abnormal synergy with each element in gait. Therefore, the influence of abnormal synergy on each factor in gait remains unknown.

\section{Conclusion}

We found that the amount of secondarily generated plantar flexion torque (STo) was as large as the selectively generated plantar flexion torque (PTo), and that the STo was negatively correlated to TUG speed. This suggests that torque generation as abnormal synergy may help for patients after stroke who cannot sufficiently generate selective torque.

\section{Abbreviations \\ PTo: The primary torque; STo: The secondary torque; TUG: Timed-up-and go test; EMG: Electromyographic.}

\section{Competing interests}

The author(s) declare that they have no competing interests.

\section{Authors' contributions}

KS designed and carried out the study, collected and analyzed the data, and drafted the manuscript. KO conceived of the study and assisted with the collection of data and the analysis of the data. Kl and YS assisted with the collection of data. TY developed orthosis-type device and collected participants. $\mathrm{SI}$ assisted in drafting the manuscript. NI designed the study, revised the manuscript critically, and gave the final approval of the manuscript. All authors have read and approved the final manuscript.

\section{Acknowledgements}

This work was partially supported by a Grant-in-Aid for Japan Society for the Promotion of Science Fellows. We thank the subjects who volunteered to participate in this study.

\section{Author details}

'Department of Physical Therapy, Human Health Sciences, Graduate School of Medicine, Kyoto University, 53, Kawahara-cho, Shogoin, Sakyo-ku, Kyoto 606-8507, Japan. ${ }^{2} J a p a n$ Society for the Promotion of Science, 5-3-1 Kojimachi, Chiyoda-ku, Tokyo 102-0083, Japan. ${ }^{3}$ Department of Rehabilitation, Biwako Gakuen Medical and Welfare Center, Kusatsu, 8-3-113 Kasayama, Kusatsu-shi, Shiga 525-0072, Japan. ${ }^{4}$ Department of Rehabilitation,

Hakuhoukai Tagawashinsei Hospital, 3638 Ooazanatsuyoshi, Tagawa-shi, Fukuoka 825-0004, Japan. ${ }^{5}$ Manufacturing Technology Section, Kawamura Gishi Co., Ltd, 1-12-1 Goryo, Daito-shi, Osaka 574-0064, Japan. 
Received: 11 June 2014 Accepted: 18 September 2014

Published: 25 September 2014

\section{References}

1. Norrving B, Kissela B: The global burden of stroke and need for a continuum of care. Neurology 2013, 80:S5-S12.

2. Dobkin $\mathrm{BH}$ : Impairments, disabilities, and bases for neurological rehabilitation after stroke. J Stroke Cerebrovasc Dis 1997, 6:221-226.

3. Nadeau S, Arsenault AB, Gravel D, Bourbonnais D: Analysis of the clinical factors determining natural and maximal gait speeds in adults with a stroke. Am J Phys Med Rehabil 1999, 78:123-130.

4. Mayo NE, Wood-Dauphinee S, Cote R, Durcan L, Carlton J: Activity, participation, and quality of life 6 months poststroke. Arch Phys Med Rehabil 2002, 83:1035-1042.

5. Aprile I, Piazzini DB, Bertolini C, Caliandro P, Pazzaglia C, Tonali P, Padua L: Predictive variables on disability and quality of life in stroke outpatients undergoing rehabilitation. Neurol Sci 2006, 27:40-46.

6. Flansbjer UB, Downham D, Lexell J: Knee muscle strength, gait performance, and perceived participation after stroke. Arch Phys Med Rehabil 2006, 87:974-980.

7. Patterson SL, Forrester LW, Rodgers MM, Ryan AS, Ivey FM, Sorkin JD, Macko RF: Determinants of walking function after stroke: differences by deficit severity. Arch Phys Med Rehabil 2007, 88:115-119.

8. Twitchell TE: The restoration of motor function following hemiplegia in man. Brain 1951, 74:443-480.

9. Brunnstrom S: Movement therapy in hemiplegia: a neurophysiological approach. New York, NY: Harper \& Row; 1970:7-33.

10. Levin MF: Interjoint coordination during pointing movements is disrupted in spastic hemiparesis. Brain 1996, 119:281-293.

11. Chen CL, Wong MK, Chen HC, Cheng PT, Tang FT: Correlation of polyelectromyographic patterns and clinical upper motor neuron syndrome in hemiplegic stroke patients. Arch Phys Med Rehabil 2000 81:869-875.

12. Beer RF, Dewald JPA, Rymer WZ: Deficits in the coordination of multijoint arm movements in patients with hemiparesis: evidence for disturbed control of limb dynamics. Exp Brain Res 2000, 131:305-319.

13. Neckel N, Pelliccio M, Nichols D, Hidler J: Quantification of functional weakness and abnormal synergy patterns in the lower limb of individuals with chronic stroke. J Neuroeng Rehabil 20 2006, 3:17.

14. Cruz TH, Dhaher YY: Evidence of abnormal lower-limb torque coupling after stroke: an isometric study. Stroke 2008, 39:139-147.

15. Hutin E, Pradon D, Barbier F, Gracies JM, Bussel B, Roche N: Lower limb coordination patterns in hemiparetic gait: factors of knee flexion impairment. Clin Biomech (Bristol, Avon) 2011, 26:304-311.

16. Barela JA, Whitall J, Black P, Clark JE: An examination of constraints affecting the intralimb coordination of hemiparetic gait. Hum Mov SCl 2000, 19:251-273.

17. Rinaldi LA, Monaco V: Spatio-temporal parameters and intralimb coordination patterns describing hemiparetic locomotion at controlled speed. J Neuroeng Rehabil 2013, 10:9.

18. Kwakkel G, Wagenaar RC: Effect of duration of upper- and lower-extremity rehabilitation sessions and walking speed on recovery of interlimb coordination in hemiplegic gait. Phys Ther 2002, 82:432-448.

19. Chen $\mathrm{CL}$, Chen HC, Tang SFT, Wu CY, Cheng PT, Hong WH: Gait performance with compensatory adaptations in stroke patients with different degrees of motor recovery. Am J Phys Med Rehabil 2003, 82:925-935.

20. von Schroeder HP, Coutts RD, Lyden PD, Billings E Jr, Nickel VL: Gait parameters following stroke: a practical assessment. J Rehabil Res Dev 1995, 32:25-31.

21. Huitema RB, Hof AL, Mulder T, Brouwer WH, Dekker R, Postema K: Functional recovery of gait and joint kinematics after right hemispheric stroke. Arch Phys Med Rehabil 2004, 85:1982-1988.

22. Allen $J$, Kautz SA, Neptune RR: Step length asymmetry is representative of compensatory mechanisms used in post-stroke hemiparetic walking Gait Posture 2011, 33:538-543.

23. Bowden MG, Clark DJ, Kautz SA: Evaluation of abnormal synergy patterns poststroke: relationship of the Fugl-Meyer Assessment to hemiparetic locomotion. Neurorehabil Neural Repair 2010, 24:328-337.

24. Neckel ND, Blonien N, Nichols D, Hidler J: Abnormal joint torque patterns exhibited by chronic stroke subjects while walking with a prescribed physiological gait pattern. J Neuroeng Rehabil 2008, 5:19.
25. Sulzer JS, Gordon KE, Dhaher YY, Peshkin MA, Patton JL: Preswing knee flexion assistance is coupled with hip abduction in people with stiffknee gait after stroke. Stroke 2010, 41:1709-1714.

26. Kim CM, Eng JJ: Magnitude and pattern of 3D kinematic and kinetic gait profiles in persons with stroke: relationship to walking speed. Gait Posture 2004, 20:140-146.

27. Parvataneni K, Olney SJ, Brouwer B: Changes in muscle group work associated with changes in gait speed of persons with stroke. Clin Biomech (Bristol, Avon) 2007, 22:813-820

28. Beer RF, Given JD, Dewald JP: Task-dependent weakness at the elbow in patients with hemiparesis. Arch Phys Med Rehabil 1999, 80:766-772.

29. Dewald JP, Beer RF: Abnormal joint torque patterns in the paretic upper limb of subjects with hemiparesis. Muscle Nerve 2001, 24:273-283.

30. Ohata K, Yasui T, Tsuboyama T, Ichihashi N: Effects of an ankle-foot orthosis with oil damper on muscle activity in adults after stroke. Gait Posture 2011, 33:102-107.

31. Gemperline JJ, Allen S, Walk D, Rymer WZ: Characteristics of motor unit discharge in subjects with hemiparesis. Muscle Nerve 1995, 18:1101-1114.

32. Hara $Y$, Masakado $Y$, Chino N: The physiological functional loss of single thenar motor units in the stroke patients: when does it occur? Does it progress? Clin Neurophysiol 2004, 115:97-103.

33. Lum PS, Burgar CG, Shor PC: Evidence for strength imbalances as a significant contributor to abnormal synergies in hemiparetic subjects. Muscle Nerve 2003, 27:211-221.

34. Gerachshenko T, Rymer WZ, Stinear JW: Abnormal corticomotor excitability assessed in biceps brachii preceding pronator contraction post-stroke. Clin Neurophysiol 2008, 119:683-692.

35. Yao J, Chen A, Carmona C, Dewald JP: Cortical overlap of joint representations contributes to the loss of independent joint control following stroke. Neuroimage 2009, 45:490-499.

36. Krishnan C, Dhaher Y: Corticospinal responses of quadriceps are abnormally coupled with hip adductors in chronic stroke survivors. Exp Neurol 2012, 233:400-407

37. Daubney ME, Culham EG: Lower-extremity muscle force and balance performance in adults aged 65 years and older. Phys Ther 1999, 79:1177-1185.

38. Janssen WGM, Bussmann HBJ, Stam HJ: Determinants of the sit-to-stand movement: a review. Phys Ther 2002, 82:866-879.

doi:10.1186/1743-0003-11-141

Cite this article as: Sakuma et al:: Relation between abnormal synergy and gait in patients after stroke. Journal of NeuroEngineering and Rehabilitation 2014 11:141.

\section{Submit your next manuscript to BioMed Central and take full advantage of:}

- Convenient online submission

- Thorough peer review

- No space constraints or color figure charges

- Immediate publication on acceptance

- Inclusion in PubMed, CAS, Scopus and Google Scholar

- Research which is freely available for redistribution 